\title{
Advancing Scientific Computation by Improving Scientific Code Development: Symbolic Execution and Semantic Analysis
}

\author{
Mark Stewart \\ QSS Group Inc. at NASA Glenn Research Center \\ Brook Park, Ohio, USA 44135 \\ Mark.E.Stewart@grc.nasa.gov
}

\begin{abstract}
This paper presents an implementation of a technique for automated, rigorous scientific program comprehension and error detection. The procedure analyzes fundamental semantic concepts during a symbolic execution of a user's code. Since program execution is symbolic, the analysis is general and can replace many test cases. The prototype of this procedure is demonstrated on two test cases including a $5 \mathrm{k}$ line of code (LOC) program. Although this technique promises a powerful tool, several challenges remain.
\end{abstract}

\section{Introduction}

As scientific computing matures, a lingering problem is the manual nature of code development and testing. Arguably, the underlying issue is code semantics - the what, why, and how of computer code - and how to automate recognition of code (perhaps even synthesis) thereby reducing the time and effort of code development, testing, and maintenance. Certainly, code developers can manually translate between their code and classical mathematical and physical formulae and concepts (with reasonable reliability). However, formalization and automation of this process has not occurred.

To address these concerns, this paper reports on an effort to formalize and automatically analyze these scientific code semantics using symbolic execution and semantic analysis.

The thesis of symbolic execution is that the semantics of a programming language's construct (the variables, data structures, and operators) can be faithfully represented symbolically. Further, this symbolic representation can be propagated during an execution of the code to provide a general and rigorous analysis.

However, symbolic execution of program statements generates symbolic expressions that will grow exponentially-unless simplified. Here semantic analysis simplifies these expressions by recognizing formulae and concepts. Semantic analysis stores and recognizes the classical mathematical, logical, and physical concepts and notation that code developers and engineers are familiar with.

The concept of symbolic execution was introduced by King [1] in 1976. In a review article, Coward [2] suggests symbolic execution has languished due to the difficulty of implementation, and cites four problems: 
1) evaluating array references dependent on symbolic values,

2) the evaluation of loops where the number of iterations is unknown,

3 ) checking the feasibility of paths: how to process branch conditions dependent on symbolic expressions,

4) how to process module calls: symbolically execute each call or execute once and abstract,

Code semantics have been the focus of some work $[3,4,5]$ including the use of an ontology and parsers for natural language understanding [6]. Petty [7] presents an impressive procedure where-during numerical execution - the units of variables and array elements are analyzed. The procedure can be easily applied to a user's code; however the numerical execution results in high wall-time and memory requirements.

This symbolic execution / semantic analysis procedure is not only intellectually appealing as a formalism; it can be fast and very general! Human programmers analyze code at approximately 0.5 LOC per minute; symbolic execution runs quickly-approximately 1000 times faster than a human-often faster than numerically executing the code itself! The procedure is general and rigorous because it uses the abstraction of symbols - not numbers; a single symbolic analysis can replace testing with a suite of conventional test cases. In Code 1, for example, if the search fails, a memory bounds error occurs. Symbolic execution detected this error, but numerical execution would require a specific set of inputs before this error occurred.

Code 1: Analysis detects how the search failure results in a memory access error

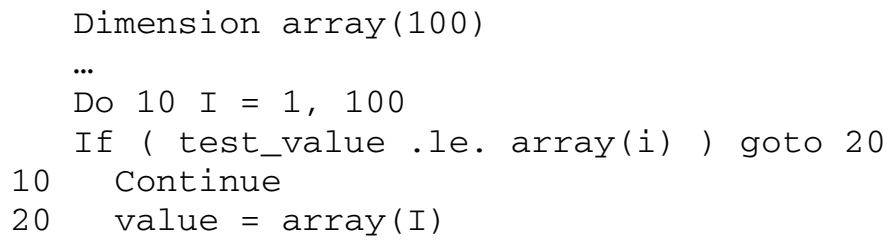

Symbolic execution / semantic analysis is not without challenges, and in the following sections, the symbolic execution procedure is explained, key problems and solutions are presented, and results are demonstrated including results for a $5 \mathrm{k}$ LOC scientific code.

\section{Symbolic Execution/Semantic Analysis Procedure}

This symbolic execution / semantic analysis procedure has two preliminary steps. First, the user makes semantic declarations (1) that provide the fundamental semantic identity of primitive variables in the code including any program inputs.

$$
\begin{aligned}
& \mathrm{A}<=\text { acceleration, } \mathrm{m} / \mathrm{s} 2 ; \\
& \mathrm{M}<=\text { mass, } \mathrm{kg} ;
\end{aligned}
$$

Second, a parser converts the user's code and semantic declarations into a tree representation in a language independent form. Symbolic execution / semantic analysis start from this basis. 


\subsection{Symbolic Execution}

Symbolic execution is similar to the ubiquitous numerical execution. In both cases statements from the user's program are executed. However, instead of loading into memory numerical values of variables, a symbolic execution emulator uses symbolic values that describe the variables. This emulator takes statements from the user's program, performs the operations on these symbols, and generates symbolic expressions. This symbolic execution emulator has a prescribed action or response to each operation encountered in a user's program, including $+,-, *, /$, **, array references, logical operators and loops. Table 1 contrasts numerical and symbolic execution.

Table 1. Comparison of numerical and symbolic execution for code statements. For numerical execution, the input file contains "4 5"; the semantic declarations are (1)

\begin{tabular}{|c|c|c|}
\hline $\begin{array}{l}\text { Code } \\
\text { Statement }\end{array}$ & $\begin{array}{l}\text { Numerical } \\
\text { Execution }\end{array}$ & $\begin{array}{c}\text { Symbolic Execution/ Semantic } \\
\text { Analysis }\end{array}$ \\
\hline READ M, A & $\begin{array}{c}\text { Place } 4 \text { into } M \text {, } \\
5 \text { into } A\end{array}$ & $\begin{array}{l}\text { Attach Semantic Declaration to } \\
\text { Instance of } M \text { and } A ; \\
\text { Ignore input file }\end{array}$ \\
\hline$B=M$ & $\begin{array}{l}\text { Transfer Number } \\
\text { Value }\end{array}$ & Transfer Symbolic Value \\
\hline$M * A$ & Calculate $4{ }^{*} 5$ & $\begin{array}{c}\text { Form "mass * acceleration", } \\
\text { "kg * } \mathrm{m} / \mathrm{s} 2 \text { ", and attempt } \\
\text { simplification by semantic analysis }\end{array}$ \\
\hline $\begin{array}{l}\text { If (A.eq.5) then } \\
\qquad \begin{array}{c}B=5 \\
\text { (n) }\end{array}\end{array}$ & $\begin{array}{l}\text { A.eq. } 5 \text { is True, } \\
\text { so } \\
\text { Transfer } 5 \text { to } B\end{array}$ & $\begin{array}{c}\text { Form "A.EQ.5 } \Rightarrow 5 \text { । B" } \\
\text { and attempt simplification by } \\
\text { semantic analysis }\end{array}$ \\
\hline
\end{tabular}

\subsection{Semantic Analysis}

As statements are symbolically executed, the generated symbolic expressions become larger-unless they are simplified. The role of semantic analysis is to recognize and simplify the fundamental mathematical, logical, and physical formulae used in these expressions. Here, parsers [8,9] are used to recognize formulae in expressions and simplify them. For example, the physical formula "Force = Mass * Acceleration" is one of many formulae encoded in one expert parser. If the parser examines the expressions in Table 1, it will recognize "Mass * Acceleration" and replace it with "Force". More details of how formulae are recognized in parsers are given in [10].

Semantic analysis is not only a vital simplification tool for successful symbolic execution; symbolic representations exist for many semantic aspects of scientific and engineering code, including units, dimensions, vector analysis, and physical and mathematical equations. Table 2 provides a comprehensive list. 
Table 2. Scientific semantic properties analyzed by the procedure, including sample equations and number of parsers

\begin{tabular}{|c|c|c|}
\hline Property Analyzed & Sample Equation & Parsers \\
\hline Physical Equation & force $\Leftarrow$ mass ${ }^{*}$ accel & $\mathbf{3}$ \\
\hline Math Equation & $\Delta \phi \Leftarrow \phi-\phi$ & $\mathbf{5}$ \\
\hline Logical Expression & $\phi \Leftarrow$ If $($ True $) \phi$ else $\theta$ & $\mathbf{2}$ \\
\hline Value / Interval & {$[1,50] \Leftarrow[0,49]+1$} & $\mathbf{2}$ \\
\hline Grid Location & $\phi_{\mathrm{i}} \Leftarrow \phi_{\mathrm{i}+1}+\phi_{\mathrm{i}-1}$ & $\mathbf{4}$ \\
\hline Vector Analysis & $\phi \cdot \phi \Leftarrow \phi_{\mathrm{x}}{ }^{2}+\phi_{\mathrm{y}}{ }^{2}+\phi_{\mathrm{z}}{ }^{2}$ & $\mathbf{1}$ \\
\hline Non-Dimensional & $\phi / \mathrm{A} \Leftarrow \chi / \mathrm{A}+\varphi / \mathrm{A}$ & $\mathbf{1}$ \\
\hline Dimensions & $\mathrm{L} \Leftarrow(\mathrm{L} / \mathrm{T}){ }^{*} \mathrm{~T}$ & $\mathbf{1}$ \\
\hline Unit & $\mathrm{m} \Leftarrow \mathrm{m} / \mathrm{s}^{*} \mathrm{~s}$ & $\mathbf{1}$ \\
\hline Object & fluid $\Leftarrow$ fluid ${ }^{*}$ anything & $\mathbf{1}$ \\
\hline Data Type & Real $\Leftarrow$ Real ${ }^{*}$ Integer & $\mathbf{1}$ \\
\hline
\end{tabular}

\section{Symbolic Execution of Array References, Loops, and Conditional Statements}

Coward [2] noted that array references, loops, and conditional expressions are a challenge in symbolic execution. The challenge is that execution of these constructs depends on the numerical value of variable(s). For example, while numerical execution of an array evaluation involves retrieving a value at a known numerical index, symbolic execution only knows what is symbolically possible for the index and must retrieve array elements within the corresponding range of index values.

Symbolic execution requires more complex analysis than for numerical execution, however advantages exist. The principle advantage is greater generality and rigorousness. The following three sections will pursue this issue for array representation, loops, and logical expressions.

\subsection{Array Assignments and References}

Code 2 demonstrates symbolic execution of a simple loop. After symbolic execution, the array $\mathrm{A}$ is represented as in Figure 1 where symbolically identical array elements - the fourth through $\mathrm{N}^{\text {th }}$ (and $2^{\text {nd }}, 3^{\text {rd }}$ and N-1 $1^{\text {st }}$ to $100^{\text {th }}$ ) - have been grouped together, while the first array element has not.

Code 2. Simple Loop shows how loops and array references are symbolically executed

$$
\begin{aligned}
& \text { Integer } A(100) \\
& \text { Read } N \\
& A(1)=5 \\
& \text { Do } 10 \quad i=4, N \\
& A(i)=1
\end{aligned}
$$

10 continue 


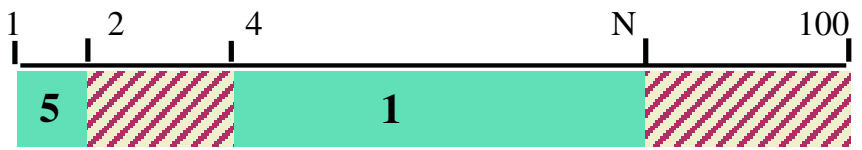

Fig. 1. Symbolic representation of the array A after execution of Code 2. Undefined values are diagonally shaded; array values are in bold; array indices are above

This grouping of symbolically identical array elements uses an ontology for array indices; the ontology entities are shown in Table 3. In Code 2, scientific code developers will easily recognize that the array variable " $\mathrm{i}$ " is a Counter, variable " $\mathrm{N}$ " is a Number.

Table 3. Entities in the Array Index Ontology. All are integer valued

\begin{tabular}{|c|c|}
\hline Index Entity & Role of Index Entity \\
\hline Integer Constant & A Known, Unchanging Value \\
\hline Number & A Variable: Unspecified, Unchanging Value \\
\hline Counter & A Variable: Taking on all Integer Values in a Range \\
\hline Compressed Counter & Scalar Representation of Counters for Multiple Array Indices \\
\hline Enumeration & A Product of Integer Constant and Number Expressions \\
\hline Offset & Delineates Multiple Arrays Stored in 1-D Array \\
\hline Offset Index & Offset plus Compressed Counter \\
\hline Index Number & A Variable: Unspecified, Unchanging Value, in a Range \\
\hline $\begin{array}{c}\text { Compressed Index } \\
\text { Number }\end{array}$ & Scalar Representation of Index Numbers for Multiple Array \\
Indices
\end{tabular}

These index entities organize an array's representation. To evaluate an array reference, the procedure compares the array indices-symbolically-with the index entities that bound the groupings in the array representation. For example, to reference $\mathrm{A}(\mathrm{N}+1)$ in Figure 1, the procedure compares $\mathrm{N}+1$ with $1,2,4, \mathrm{~N}$, and 100, and concludes $\mathrm{A}(\mathrm{N}+1)$ is in the final grouping of array entries from $\mathrm{N}+1$ to 100 .

Not only does the grouping of symbolically identical array elements use memory efficiently, it eliminates duplicate analysis. Where loops apply an identical operation over large parts of an array-as is so common in scientific computing-the semantic analysis is reduced to one analysis of an array assignment or reference. This is the principle reason why symbolic execution / semantic analysis can be faster than numerical execution of the same code!

\subsection{Loop Evaluation}

Loop evaluation is a further hurdle in symbolic execution. The issue is whether dependencies exist between loop iterations. Often no dependencies exist between iterations, and straight line symbolic execution is possible. If dependencies do exist between loop iterations, an attempt is made to use Mathematical Induction to deduce values. 


\subsection{Conditional Expressions}

Symbolic execution of conditional expressions is a challenging issue since symbolic values in the condition force the examination of each block of conditional code. The current procedure symbolically executes the statements from each possible code block. Then, for each variable, the procedure forms a conditional expression that is valid following the conditional expression. This expression is propagated through the following code.

\subsection{Subroutine Calls}

The current procedure deals with routine calls by symbolically transferring call line parameters and global variables to a child symbol table, and then symbolically executing the routine. Upon completion of the routine, call line parameters and global variables are updated in the parent symbol table.

In the test cases studied, repeat calls to routines were not excessive-since routine calls within loops are executed once or a few times. In principle, symbolically identical routine calls need not be repeated, but this feature has not been implemented yet.

\subsection{Speed of Symbolic Execution/Semantic Analysis}

The wall time requirements for symbolic execution of a code are fundamentally different from the wall time requirements of numerical execution. Two opposing issues influence the wall time — and decide the economics—of symbolic execution.

First, symbolic execution is considerably more expensive than numerical execution on an operation by operation basis. Numerical execution of " $A * B$ " includes memory accesses and a floating point operation - usually within optimized software and hardware. Symbolic execution of "A*B" includes constructing a data structure representation of the expression, and its examination by several expert parsers.

Second, the computationally demanding parts of scientific codes are usually the iterations of code within loops. Yet, in symbolic execution of a loop, symbolically equivalent (and numerically different) iterations can be grouped together and analyzed once. Consequently, symbolic execution is very attractive for loop intensive code. In particular, symbolic execution can be faster than symbolic execution for loop intensive code. Conversely, codes with fewer loops can execute more slowly symbolically than numerically.

\section{Demonstration of Results}

This symbolic execution / semantic analysis procedure has been developed and tested with two codes.

COMDES is a 1-dimensional aerodynamic design code written in FORTRAN77 with extensive use of aerodynamic formulae, relatively less use of mathematical formulae, and minimal use of subroutines. Symbolic execution completes successfully with $100 \%$ semantic analysis of units (Table 4).

STAGE2 is a 5k LOC, 2-dimensional computational fluid dynamics (CFD) code that solves turbulent, aerodynamic flow over compressor blade sections. Written in 
FORTRAN77, it is aggressively coded and makes extensive use of mathematical formulae, loops, array references and assignments (compacting multi-dimensional arrays into a 1D array, and multiple blocks of data into a 1D array), conditional expressions, and routine calls. The symbolic execution and semantic analysis of units completes almost completely. Details are shown in Table 4. For realistic grids and number of iterations, the resulting loop sizes make symbolic execution much faster than conventional numerical execution.

Table 4. Current performance results for the semantic analysis program's analysis of two test cases. Max. memory is the gross memory required to represent and retain all local and global semantic information during the semantic analysis; the executable size is 5.0 MByte. Calculations performed on a PC with a Pentium $42.2 \mathrm{GHz}$ processor with 512 MByte of RAM. The analysis results reflect the semantic analysis code's quality and not the quality or ability of the tested codes

\begin{tabular}{|c|c|c|c|c|c|c|}
\hline Code & $\begin{array}{c}\text { Lines (k } \\
\text { loc) }\end{array}$ & $\begin{array}{c}\text { Semantic } \\
\text { Declarations }\end{array}$ & $\begin{array}{c}\text { Symb Exec Wall } \\
\text { Time (s) }\end{array}$ & $\begin{array}{c}\text { Unit } \\
\text { Recognition } \\
\text { Rate (\%) }\end{array}$ & $\begin{array}{c}\text { Statements } \\
\text { Executed (k) }\end{array}$ & $\begin{array}{c}\text { Max } \\
\text { Memory } \\
\text { (MBytes) }\end{array}$ \\
\hline Comdes & 0.4 & 42 & 15.1 & 100. & .5 & 5.5 \\
\hline STAGE2 & 4.9 & 87 & 199.4 & 93.9 & 8.2 & 65.3 \\
\hline
\end{tabular}

\section{Discussion}

\subsection{Semantic Complexity}

Refinement of this symbolic execution / semantic analysis procedure revealed increasingly complex semantic concepts corresponding to aggressive programming techniques. For example, array indices were encountered that store a multi-dimensional array in a 1D array, and store multiple blocks of data in a 1D array.

This observation poses several questions: "Is the population of semantic concepts used in code limited or bounded?", "Does clear, well written code use only a bounded set of basic semantic concepts?", and "What are the limits on human programmers' knowledge, comprehension, and reliability?"

\subsection{Inference Chains and Recognition Reliability}

Reliability is a big challenge for symbolic execution. Recognizing and simplifying one expression depends on successfully recognizing and simplifying preceding expressions; conversely, failing to recognize one result usually prevents any further recognition. For example, in Code 2, a failure to locate and assign to $\mathrm{A}(1)$ compromises the remainder of the analysis.

This dependency is called the inference chain or inference tree. A code's inference chains can be exceedingly long. In COMDES, chains as long as 140 inferences have been measured, and the longest chains in STAGE2 are probably at least an order of magnitude greater. As code size and number of inferences increase, the chance of a recognition failure also increases and reliability decreases. 


\subsection{Problem Difficulty}

The difficulty of implementing symbolic execution / semantic analysis is a further challenge. Complete symbolic execution of the STAGE2 code involved previous work [10] plus a large extension effort that was completed (part-time) over 3 years. Achieving symbolic execution of the next code is easier, but still a major effort. The expectation is that refinement efforts accumulate so that development time drops for each successive user's code until closure is reached.

\section{Conclusions}

This work demonstrates that rigorous, automated symbolic analysis of scientific code is possible with a formalization of code semantics. Further, this work reveals the challenges of symbolic execution / semantic analysis, in particular, semantic complexity and recognition reliability.

Lastly, this work emphasizes the fundamental role of semantics in software, and how this role has been obscured by our contentment with manual software development.

\section{References}

1. King, J. C., "Symbolic Execution and Program Testing," Communications of ACM, 1976, 19, (7), pp. 385-394

2. Coward, P. D., "Symbolic Execution Systems-A Review," Software Engineering Journal, Nov. 1988. P 229-239

3. L. M. Wills, “Automated Program Recognition: A Feasibility Demonstration," Artificial Intelligence 45 (1-2): 113-172 (1990).

4. A. Quilici, "A Memory-Based Approach to Recognizing Programming Plans," Comm. of the ACM, 37(5):84 (1994).

5. B. Di Martino, C. W. Keßler, "Two Program Comprehension Tools for Automatic Parallelization," IEEE Concurrency, Jan.-March 2000.

6. J. Allen, Natural Language Understanding (Benjamin/Cummings, Menlo Park, 1987)

7. Petty, G. W., "Automated computation and consistency checking of physical dimensions and units in scientific programs," Software-Practice and Experience, 2001; 31 pp.1067-1076

8. S. C. Johnson, "Yacc--Yet Another Compiler-Compiler," Comp. Sci. Tech. Rep. No. 32. (AT\&T Bell Laboratories, Murray Hill, 1977).

9. A.V. Aho, R. Sethi, J. D. Ullman, Compilers: Principles, Techniques, and Tools (Addison-Wesley, Reading, 1986).

10. M.E.M. Stewart, S. Townsend, "An Experiment in Automated, Scientific-Code Semantic Analysis," AIAA-99-3276, June 1999 